\title{
Metric Bases for Polyhedral Gauges
}

\author{
Fabien Rebatel and Édouard Thiel \\ Laboratoire d'Informatique Fondamentale de Marseille (LIF, UMR 6166), \\ Aix-Marseille Université, \\ 163 Avenue de Luminy, Case 901, 13288 Marseille cedex 9, France \\ \{Fabien.Rebatel, Edouard.Thiel\}@lif.univ-mrs.fr
}

\begin{abstract}
Let $(W, d)$ be a metric space. A subset $S \subseteq W$ is a resolving set for $W$ if $d(x, p)=d(y, p)$ for all $p \in S$ implies $x=y$. A metric basis is a resolving set of minimal cardinality, named the metric dimension (of $W$ ). Metric bases and dimensions have been extensively studied for graphs with the intrinsic distance, as well as in the digital plane with the city-block and chessboard distances. We investigate these concepts for polyhedral gauges, which generalize in the Euclidean space the chamfer norms in the digital space.
\end{abstract}

Keywords: metric basis, metric dimension, resolving set, polyhedral gauge, chamfer norms, discrete distance, distance geometry.

\section{Introduction}

Distance geometry is the characterization and study of sets of points based on the distance values between member pairs. A general program is laid out in 2. We investigate in this paper the classical notions of metric dimension and metric basis in the context of digital geometry, for the class of polyhedral distance gauges which generalize the chamfer (or weighted) norms.

Let $W$ be a set endowed with a metric $d$, and take $S=\left(p_{1}, p_{2}, \ldots, p_{k}\right)$ an ordered subset of elements in $W$. These elements are usually called points, vertices, anchors or landmarks in the literature. The representation of $q \in W$ with respect to $S$ is the $k$-tuple $r(q \mid S)=\left\{d\left(p_{1}, q\right), d\left(p_{2}, q\right), \ldots, d\left(p_{k}, q\right)\right\}$, also called the $S$-coordinates of $q$. The set $S$ is a resolving set (or locating set) for $W$ if every two elements of $W$ have distinct representation. The metric dimension (or simply dimension, or location number) $\operatorname{dim}(W)$ is the minimum cardinality of a resolving set for $W$. A resolving set having minimal cardinality is called a metric basis (or reference set) for $W$.

The metric dimension has been extensively studied in graph theory. Using the intrinsic metric, Harary and Melter gave in [9] the metric dimension of any path, complete graph, cycle, wheel and complete bipartite graph, and proposed an algorithm for finding a metric basis of a tree $T$, which gives an explicit formula for the dimension of $T$.

Khuller et al. showed in [13] that the intrinsic metric dimension of trees can be efficiently solved in linear time. All graphs having dimension 1 are paths. 
A graph having dimension 2 cannot have the complete 5 -clique $K_{5}$ nor the complete bipartite graph $K_{3,3}$ (see [19]) as a subgraph. However, there are nonplanar graphs having dimension 2 . The dimension of an arbitrary graph with $n$ nodes can be approximated within a factor $O(\log n)$ in polynomial time. Finally, finding the metric dimension of an arbitrary graph is NP-hard.

In [5], Chartrand et al. presented bounds of the dimension of a graph $G$ in terms of the order and the diameter of $G$, and determined all connected graphs of order $n$ having dimension $n-1$ or $n-2$. They also showed how to find the metric dimension and a basis for a graph in terms of an integer programming problem. A collection of bounds or exact values of metric dimensions is summarized in [1], for several families of connected graphs as well as for the join and the Cartesian product of graphs. The dimension of a graph with an added vertex is studied in [3]. For results on infinite locally finite graphs, see 4].

In digital geometry, the notion of metric dimension is equally natural. Melter and Tomescu showed in 14 the following results. The metric bases for the digital plane with Euclidean distance $d_{2}$ consist precisely of sets of three non-collinear points, whereas with respect to the city block distance $d_{1}$ and the chessboard distance $d_{\infty}$, the digital plane has no finite metric bases. From the point of view of applications, only finite sets are of interest, mainly rectangular regions. In the case of axes-parallel rectangles, the $d_{1}$ metric dimension of a rectangle is 2 , and the $d_{\infty}$ metric dimension of a square is 3 . If non axes-parallel rectangles are considered, the situation is less simple: for both distances, there exists a rectangle in the digital plane such that its dimension is $n$, for any given $n \geqslant 3$. In [13, Khuller et al. proved that the $d_{1}$ metric dimension of a $n$-dimensional axes-parallel rectangle is $n$.

Several refinements of these bases notions have been proposed. Chartrand and Zhang defined and studied in [8] the notions of forcing subset, number and dimension. A subset $F$ of a basis $S$ of $W$ is a forcing subset of $S$ if $S$ is the unique basis of $W$ containing $F$. The forcing number is the minimum cardinality of a forcing subset for $S$, while the forcing dimension is the minimum forcing number among all bases of $W$. The forcing concepts have been studied for various subjects, see 8 for references.

Another interesting notion is the partition dimension, proposed in [6] [7. Let $\Pi=\left(S_{1}, \ldots, S_{k}\right)$ be an ordered $k$-partition of $W$, and consider the distance to a set $d(p, S)=\min \{d(p, q): q \in S\}$ where $p \in W$ and $S \subset W$. The representation of $p$ with respect to $\Pi$ is $r(p \mid \Pi)=\left\{d\left(p, S_{1}\right), \ldots, d\left(p, S_{k}\right)\right\}$. The partition dimension $\operatorname{pd}(W)$ and partition bases are then defined in the same manner as the metric ones. It is shown that for any nontrivial connected graph $G$ we have $\operatorname{pd}(G) \leqslant \operatorname{dim}(G)+1[6]$. Tomescu showed in [18] that the partition dimension may be much smaller than the metric dimension. In particular, the partition dimension of the digital plane with respect to $d_{1}$ and $d_{\infty}$ is 3 and 4 , respectively.

These concepts have important applications. In fact, distance geometry has immediate relevance where distance values are considered, such as in cartography, physics, biology, chemistry, classification, combinatorial search and optimization, game theory, and image analysis. For instance, locating and reference 
sets are useful when working with sonar and LORAN stations for navigation [16]. In robot motion planing, minimizing a set of landmarks uniquely determine a robot's position 13 . A chemical compound is frequently viewed as a labeled graph, for which a metric basis allows to determine functional groups [3].

We are here mainly concerned by image analysis and digital geometry. While the metric bases and dimension concepts have been studied in [14 for $d_{1}$ and $d_{\infty}$, there is up to our knowledge no study available for an important class of digital metrics, widely used in image analysis: the chamfer distances. We aim at studying these metric concepts for these distances, and more generally for the class of polyhedral distance gauges, coming from Euclidean geometry.

The paper is organized as follows. In section 2 we recall the classical definitions of metric, norm and gauge, we state additional properties of gauges, and describe the link between gauges and chamfer norms. In section 3, we show that polyhedral gauges do not have finite metric bases in $\mathbb{R}^{n}$. Then, in section 4, we take a look at some classes of gauges having small metric dimension in a rectangle. Next in section 5 we present some experimental results. We finally conclude in section 6 .

\section{Norms and Gauges}

\subsection{Preliminaries}

We first recall some classical definitions. A metric $d$ on a nonempty set $W$ is a function $d: W \times W \rightarrow \mathbb{R}$ which is positive defined, symmetric and sub-additive (the triangle inequality holds). A set $W$ associated to a metric $d$ is called a metric space, denoted by $(W, d)$.

Given a metric space $(W, d)$, the (closed) ball of center $p \in W$ and radius $r \in \mathbb{R}$ is the set $\mathcal{B}(p, r)=\{q \in W: d(p, q) \leqslant r\}$.

To define a norm, we need the notion of vector space, which we don't have in a graph, but is natural in $\mathbb{R}^{n}$ and can be extended in $\mathbb{Z}^{n}$. A vector space is a set of vectors defined over a field. The generalization of the notion of a vector space over a ring $A$ for a set $W$ is named a module, denoted $(W, A)$. Each module can be associated to an affine space, and reciprocally. Since $\mathbb{Z}^{n}$ is a module over $\mathbb{Z}$, this concept allows us to properly define a norm on $\mathbb{Z}^{n}$.

A norm $g$ on a module $(W, A)$ with values in $\mathbb{R}$ is a function $g: W \rightarrow \mathbb{R}$ which is positive defined, homogeneous in $A$ and sub-additive. Each norm induces a metric. A metric $d$ is associated to a norm $g$ if and only if $d$ is translation invariant and homogeneous in $A$.

The Minkowski distance of order $p \geqslant 1$ between two points $x=\left(x_{1}, \ldots, x_{n}\right)$ and $y=\left(y_{1}, \ldots, y_{n}\right)$ is defined by $d_{p}(x, y)=\left(\sum_{i=1}^{n}\left|x_{i}-y_{i}\right|^{p}\right)^{1 / p}$. It is well-known that any Minkowski distance is a metric and induces a norm (denoted $\ell_{p}$ ). The $d_{1}$ distance is also called Manhattan or city block distance, the $d_{\infty}$ distance is known as the chessboard distance, and $d_{2}$ is the Euclidean distance.

We call $\Sigma^{n}$ the group of axial and diagonal symmetries in $\mathbb{Z}^{n}$ about centre $O$. The cardinal of the group is $\# \Sigma^{n}=2^{n} n$ ! (which is 8,48 and 384 for $n=2$, 3 and 4). A set $S$ is said to be grid-symmetric if for every $\sigma \in \Sigma^{n}$ we have 

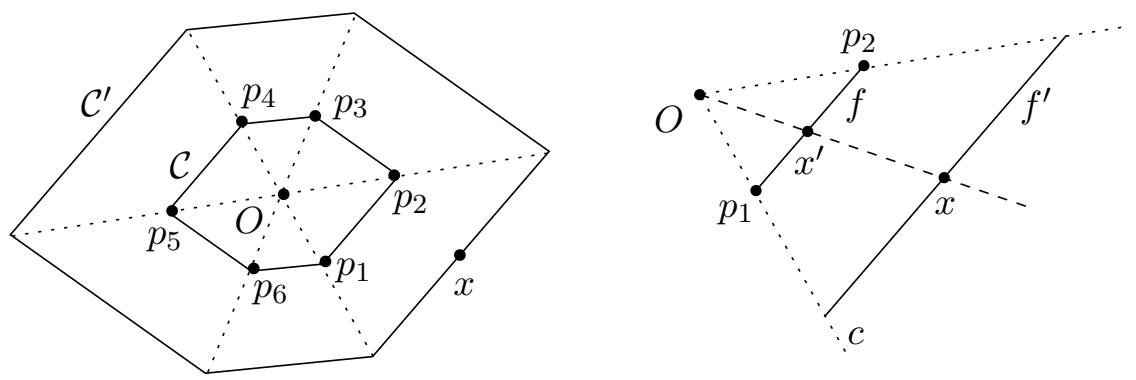

Fig. 1. Left: A gauge $\gamma_{\mathcal{C}}$, its unit ball $\mathcal{C}$, and the convex ball $\mathcal{C}^{\prime}$ scaled by $d_{\mathcal{C}}(O, x)$. Right: Illustration of the proof of lemma 1

$\sigma(S)=S$. The generator of a grid-symmetric set $S \subseteq \mathbb{Z}^{n}$ (or $\mathbb{R}^{n}$ ) is $\mathcal{G}(S)=$ $\left\{\left(p_{1}, \ldots, p_{n}\right) \in S: 0 \leqslant p_{n} \leqslant p_{n-1} \leqslant \ldots \leqslant p_{1}\right\}$. The Minkowski distance balls are grid-symmetric.

\subsection{Gauges}

Gauges are usually defined in the Euclidean space $\mathbb{R}^{n}$ and can be considered in the digital space $\mathbb{Z}^{n}$ as well.

Given a convex $\mathcal{C}$ containing the origin $O$ in its interior, a gauge for $\mathcal{C}$ is the function $\gamma_{\mathcal{C}}(x)$ defined by the minimum positive scale factor $\lambda$, necessary for having $x \in \lambda \mathcal{C}$. Formally, $\gamma_{\mathcal{C}}(x)=\inf \left\{\lambda \in \mathbb{R}_{+}: x \in \lambda \mathcal{C}\right\}$. By construction, this function is positive, homogeneous in $\mathbb{R}$ and sub-additive.

By definition, all norms are gauges for their unit ball. Conversely, a gauge for $\mathcal{C}$ is a norm, denoted $n_{\mathcal{C}}$, iff $\mathcal{C}$ is central-symmetric [1]. We call distance gauge, denoted $d_{\mathcal{C}}$, the metric induced by a central-symmetric gauge $\gamma_{\mathcal{C}}$.

Since we are concerned with norms, we will only consider central-symmetric gauges in the remainder of the paper.

We now deal with the case where $\mathcal{C}$ is a central-symmetric convex polyhedron centered in $O$. Pick a facet $f$ of $\mathcal{C}$ and denote $p_{1}, \ldots, p_{m}$ its vertices. Let $x$ be a point of the cone $c=(O, f)$ (see Fig. 1). We can express $x$ as a unique positive linear combination of the vertices: $\exists \lambda_{1}, \ldots, \lambda_{m} \in \mathbb{R}_{+}$such that $x=\sum_{i=1}^{m} \lambda_{i} p_{i}$.

Lemma 1 (Polyhedral gauge distance). $d_{\mathcal{C}}(O, x)=\sum_{i=1}^{m} \lambda_{i}$.

Proof. Let $x \in \mathcal{C} \backslash\{O\}$. There exists $\left\{\lambda_{i}\right\}_{1 \leqslant i \leqslant m}$ such that $x=\sum_{i} \lambda_{i} p_{i}$ and $\forall i, \lambda_{i} \geqslant 0$. Fix $x^{\prime}=f \cap[O x)$. Then $\exists \lambda>0$ such that $x=\lambda x^{\prime}$. Hence $x^{\prime}=$ $\frac{1}{\lambda} \sum_{i} \lambda_{i} p_{i}=\sum_{i} \frac{\lambda_{i}}{\lambda} p_{i}$. Since $x^{\prime} \in f$, we have $\sum_{i} \frac{\lambda_{i}}{\lambda}=1$. Thus $\lambda=\sum_{i} \lambda_{i}$. By definition $d_{\mathcal{C}}(O, x)=\gamma_{\mathcal{C}}(x)=\lambda \gamma_{\mathcal{C}}\left(x^{\prime}\right)$ and $\gamma_{\mathcal{C}}\left(x^{\prime}\right)=1$, therefore $d_{\mathcal{C}}(O, x)=\lambda$.

In the sequel, we denote by $g_{c}(x)$ the gauge distance $d_{\mathcal{C}}(O, x)$ in the cone $c$.

Lemma 2 (Local additivity). $\forall x, y \in c, g_{c}(x+y)=g_{c}(x)+g_{c}(y)$. 
Proof. By definition of the cone $c$ we have $c=\left\{\sum_{i} \delta_{i} p_{i}: \delta_{i} \in \mathbb{R}_{+}\right\}$. Let $x=$ $\sum_{i} \alpha_{i} p_{i}, \alpha_{i} \in \mathbb{R}_{+}$and $y=\sum_{i} \beta_{i} p_{i}, \beta_{i} \in \mathbb{R}_{+}$. Then $x+y=\sum_{i}\left(\alpha_{i} p_{i}\right)+\sum_{i}\left(\beta_{i} p_{i}\right)=$ $\sum_{i}\left(\alpha_{i}+\beta_{i}\right) p_{i}$. Since $\alpha_{i}+\beta_{i} \geqslant 0$ then $x+y \in c$. By application of lemma 1 we have $g_{c}(x)=\sum_{i} \alpha_{i}, g_{c}(y)=\sum_{i} \beta_{i}$ and $g_{c}(x+y)=\sum_{i} \alpha_{i}+\beta_{i}$.

\subsection{Chamfer Norms}

The chamfer distances have been extensively used and studied in image analysis, see [17] 12 15] for references. The $d_{1}$ and $d_{\infty}$ distances are peculiar cases of chamfer distances, and the class of chamfer norms can be seen as a special case of polyhedral gauges.

Here we recall some results from [17, §4.2-4.3]. A chamfer mask $\mathcal{M}$ in $\mathbb{Z}^{n}$ is a central-symmetric set $\mathcal{M}=\left\{\left(\overrightarrow{v_{i}}, w_{i}\right) \in \mathbb{Z}^{n} \times \mathbb{Z}_{+*}\right\}_{1 \leqslant i \leqslant m}$ containing at least a basis of $\mathbb{Z}^{n}$, where $\left(\overrightarrow{v_{i}}, w_{i}\right)$ are called weightings, $\overrightarrow{v_{i}}$ vectors and $w_{i}$ weights. The chamfer distance $d_{\mathcal{M}}$ between two points $p, q \in \mathbb{Z}^{n}$ is

$$
d_{\mathcal{M}}(p, q)=\min \left\{\sum \lambda_{i} w_{i}: \sum \lambda_{i} \overrightarrow{v_{i}}=\overrightarrow{p q}, 1 \leqslant i \leqslant m, \lambda_{i} \in \mathbb{Z}_{+}\right\} .
$$

If we consider the infinite weighted graph $G_{\mathcal{M}}$ where the vertices are the points of $\mathbb{Z}^{n}$, and the edges and weights are given by the weightings of $\mathcal{M}$ translated around each point, then $d_{\mathcal{M}}$ is the intrinsic distance of $G_{\mathcal{M}}$, hence $d_{\mathcal{M}}$ is a metric.

Let $\mathcal{M}^{\prime}=\left\{O+\overrightarrow{v_{i}} / w_{i}\right\}_{1 \leqslant i \leqslant m} \in \mathbb{R}^{n}$ and let $B_{\mathcal{M}}^{\prime}=\operatorname{conv}\left(\mathcal{M}^{\prime}\right)$, then $B_{\mathcal{M}}^{\prime}$ is a central-symmetric and convex polyhedron whose facets separate $\mathbb{R}^{n}$ in cones from $O$. A facet $\mathcal{F}$ of $B_{\mathcal{M}}^{\prime}$ is generated by a subset $\left.\mathcal{M}\right|_{\mathcal{F}}=\left\{\left(\overrightarrow{v_{j}}, w_{j}\right)\right\}$ of $\mathcal{M}$; if $\mathcal{F}$ is simplicial and if $\Delta_{\mathcal{F}}=\operatorname{det}\left\{\overrightarrow{v_{j}}\right\}$ is such that $\left|\Delta_{\mathcal{F}}\right|=1$, then $\mathcal{F}$ is said unimodular.

In the Euclidean space $\mathbb{R}^{n}$, we can define an analytic continuation $d_{\mathcal{M}}^{\mathbb{R}}$ of $d_{\mathcal{M}}$ by replacing $\lambda_{i} \in \mathbb{Z}_{+}$with $\lambda_{i} \in \mathbb{R}_{+}$in (1). It is easy to see that $d_{\mathcal{M}}^{\mathbb{R}}$ is the distance gauge for $B_{\mathcal{M}}^{\prime}$, thus $d_{\mathcal{M}}^{\mathbb{R}}$ is always a norm; while in $\mathbb{Z}^{n}$, the following norm condition has to be fulfilled: $d_{\mathcal{M}}$ is a norm in $\mathbb{Z}^{n}$ if and only there exists a unimodular triangulation of the facets of $B_{\mathcal{M}}^{\prime}$ (see [17, p. 53] and [12, §6.3], or an equivalent condition in $[15, \S 3.3])$. This condition garanties that $d_{\mathcal{M}}$ in $\mathbb{Z}^{n}$ is the Gauss discretization of $d_{\mathcal{M}}^{\mathbb{R}}$ in $\mathbb{R}^{n}$.

Now let $d_{\mathcal{M}}$ be a chamfer norm, $\mathcal{F}$ a simplicial facet of $B_{\mathcal{M}}^{\prime}$ and $\left.\mathcal{M}\right|_{\mathcal{F}}=$ $\left\{\left(\overrightarrow{v_{j}}, w_{j}\right)\right\}_{1 \leqslant j \leqslant n}$; then for each point $p=\left(p_{1}, \ldots, p_{n}\right)$ in the cone $(O, \mathcal{F})$, called influence coneof $\left.\mathcal{M}\right|_{\mathcal{F}}$, we have $d_{\mathcal{M}}(O, p)=p_{1} \delta_{1}+\cdots+p_{n} \delta_{n}$, where $\left(\delta_{1}, \ldots, \delta_{n}\right)=$ $\overrightarrow{\delta_{\mathcal{F}}}$ is a normal vector of $\mathcal{F}$, and $\delta_{k}$ is the elementary displacement for the $k^{\text {th }}$ coordinate:

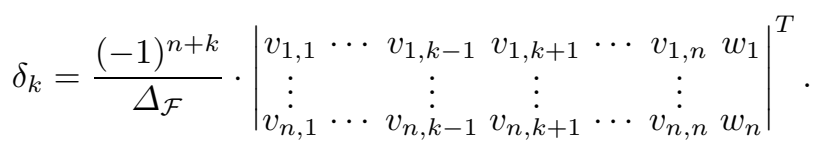

\section{Metrics Bases for Polyhedral Gauges in Infinite Space}

We show in this section that polyhedral gauges do not have finite bases in $\mathbb{R}^{n}$. 
Given a convex polyhedron $\mathcal{C}$ in $\mathbb{R}^{n}$, an hyperplane $h_{i}$ is a supporting hyperplane if $h_{i}$ contains a facet $f_{i}$ of $\mathcal{C}$. We name supporting half-space $H_{i}$ the half-space bounded by $h_{i}$ and containing $\mathcal{C}$. The intersection $\cap_{i} H_{i}$ of the supporting half-spaces is equal to $\mathcal{C}$. In $\mathbb{R}^{n}$, the decomposition of $\mathcal{C}$ in supporting half-spaces is unique. Note that this is generally not the case in $\mathbb{Z}^{n}$, see [15].

Let $\left\{H_{i}\right\}$ be the representation in half-spaces of a convex polyhedron $\mathcal{C} \in$ $\mathbb{R}^{n}$ having nonempty interior, and $\left\{h_{i}\right\}$ the corresponding supporting hyperplanes. If the intersection $\cap_{i} h_{i}$ is a single point $a$, then $\mathcal{C}$ is a polyhedral cone and $a$ is called its apex. For convenience, this cone is denoted by $\left(a,\left\{H_{i}\right\}\right)$. By construction, a polyhedral cone is unbounded. We name $\Lambda$ the set of the polyhedral cones in $\mathbb{R}^{n}$ having non-empty interior. A ray stands for a half-line.

Lemma 3. For each cone $c=\left(a,\left\{H_{i}\right\}\right) \in \Lambda$, it always exists a point $p \in c$ and a ray $]$ ap $) \subset c$ that does not belong to any facet of $c$.

Proof. $c$ is nonempty by definition of $\Lambda$, so an interior point $p$ always exists. Let us prove that $p \notin \cup_{i} h_{i} \Rightarrow \forall \lambda>0, a+\lambda p \notin \cup_{i} h_{i}$. Suppose that $\exists \lambda_{0}>0$ such that $q=a+\lambda_{0} p \in \cup_{i} h_{i}$. Then there is at least one $i_{0}$ such that $q \in h_{i_{0}}$, hence $(a q) \in h_{i_{0}}$, but $p \in(a q)$ so $p \in h_{i_{0}}$, a contradiction.

We now translate some supporting half-planes. Let us consider a cone $c=$ $\left(a,\left\{H_{i}\right\}_{1 \leqslant i \leqslant m}\right) \in \Lambda$, and a set of translations $\left\{t_{i}\right\}_{1 \leqslant i \leqslant m}$.

Lemma 4. The convex $c^{\prime}=\bigcap\left\{H_{i}^{\prime}=H_{i}+t_{i}\right\}_{1 \leqslant i \leqslant m}$ is an unbounded nonempty convex polyhedron.

Proof. Given an interior point $p \in c$, we consider the line $L=(a p)$. For each $1 \leqslant i \leqslant m$, we have $p \notin h_{i}$ so $L$ intersects $h_{i}$ in the single point $a$. Since $p \in H_{i}$, $L \cap H_{i}$ is the ray $[a p)$. Using the same argument, we deduce that $L \cap H_{i}^{\prime}$ is a ray. Since $c^{\prime}=\cap_{i} H_{i}^{\prime}$ we have $c^{\prime} \cap L=\left(\cap_{i} H_{i}^{\prime}\right) \cap L=\cap_{i}\left(H_{i}^{\prime} \cap L\right)$, thus $c^{\prime} \cap L$ is the intersection of rays belonging to the same line $L$, having same orientation but having different apexes, so $c^{\prime} \cap L$ is a ray, and we can conclude that $c^{\prime}$ is unbounded and nonempty.

It is easy to see that the resulting convex is not necessarily a cone.

We have just considered a single cone and we have applied a number of translations of the half-spaces defining the cone. We will now see a property concerning several cones obtained by translations on a common starting cone. Given a cone $c=\left(a,\left\{H_{i}\right\}_{1 \leqslant i \leqslant m}\right) \in \Lambda$ and a set $\left\{t_{j}\right\}_{1 \leqslant j \leqslant k}$ of translations in $\mathbb{R}^{n}$, we define the cones $c_{1}, \ldots, c_{k}$ as $c_{j}=c+t_{j}=\left(a+t_{j},\left\{H_{i}+t_{j}\right\}_{1 \leqslant i \leqslant m}\right)$.

Lemma 5. The intersection of the cones $c_{1}$ to $c_{k}$ is an unbounded and nonempty convex polyhedron.

Proof. The intersection $\cap_{j=1}^{k}\left(c_{j}\right)$ is equal to $\cap_{j=1}^{k}\left(\cap_{i=1}^{m}\left(H_{i}+t_{j}\right)\right)$, which is equal to $\cap_{i=1}^{m}\left(\cap_{j=1}^{k}\left(H_{i}+t_{j}\right)\right)$. Now it is self-evident that each $\cap_{j=1}^{k}\left(H_{i}+t_{j}\right)$ results in a single half-space (by intersection of parallel half-spaces). More precisely, $\cap_{j=1}^{k}\left(H_{i}+t_{j}\right)=H_{i}+t_{i}^{\prime}$ where $t_{i}^{\prime} \in\left\{t_{1}, \ldots, t_{k}\right\}$. So we can rewrite $\cap_{j=1}^{k} c_{j}=$ $\cap_{i=1}^{m}\left(H_{i}+t_{i}^{\prime}\right)$. The result follows by lemma 4 . 

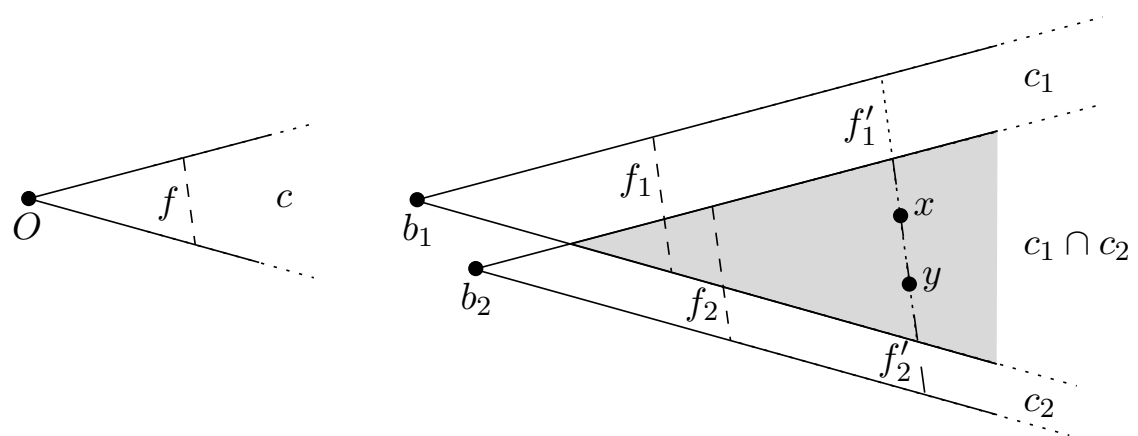

Fig. 2. On the left, the cone $c=(O, f)$. On the right, the two cones defined by $c_{1}=$ $c+\overrightarrow{O b_{1}}$ and $c_{2}=c+\overrightarrow{O b_{2}}$. In grey the intersection between $c_{1}$ and $c_{2}$. As a result, $x$ and $y$ both lie on $f_{1}^{\prime}$ and $f_{2}^{\prime}$.

The lemma 1 provides a simple formula to calculate the distance values in a polyhedral gauge. So as to compute the formula, we need to split the space into cones. Given a polyhedral gauge $\gamma_{\mathcal{C}}$ and a facet $f$ of $\mathcal{C}$, consider the cone $c=(O, f)$. Fix $b_{1}$ and $b_{2}$ two distinct points. Denote $c_{1}=c+\overrightarrow{O b_{1}}$ and $c_{2}=c+\overrightarrow{O b_{2}}$ the translated cones respectively centered in $b_{1}$ and $b_{2}$ (see Fig. (2).

Lemma 6. $\forall x, y \in c_{1} \cap c_{2}, g_{c_{1}}(x)=g_{c_{2}}(y) \Longleftrightarrow g_{c_{2}}(x)=g_{c_{2}}(y)$.

Proof. Given a cone $c=(O, f)$ and two points $b_{1}$ and $b_{2}$, we define $c_{1}=c+\overrightarrow{O b_{1}}=$ $\left(b_{1}, f_{1}\right)$ and $c_{2}=c+\overrightarrow{O b_{2}}=\left(b_{2}, f_{2}\right)$. Set $x$ a point in $c_{1} \cap c_{2}$. We name $f_{1}^{\prime}$ the facet $f_{1}$ scaled by $\lambda_{1}=d_{\mathcal{C}}\left(b_{1}, x\right)=g_{c_{1}}(x)$ and name $f_{2}^{\prime}$ the facet $f_{2}$ scaled by $\lambda_{2}=d_{\mathcal{C}}\left(b_{2}, x\right)=g_{c_{2}}(x)$. Since $f_{1}^{\prime}$ and $f_{2}^{\prime}$ are parallel and intersect $x, f_{1}^{\prime}$ and $f_{2}^{\prime}$ are in the same hyperplane. Hence, for any point $y \in f_{1}^{\prime}$, we also have $y \in f_{2}^{\prime}$. Finally, by definition of gauges, we have $g_{c_{2}}(x)=g_{c_{2}}(y)$.

Lemma 6 also holds with several points. Indeed, considering any point $b_{3}$, denote $c_{3}$ the cone defined by $c_{3}=c+\overrightarrow{O b_{3}}$. Then, by transitivity, $\forall x, y \in \cap_{i} c_{i}, g_{c_{1}}(x)=$ $g_{c_{1}}(y) \Leftrightarrow g_{c_{2}}(x)=g_{c_{2}}(y) \Leftrightarrow g_{c_{3}}(x)=g_{c_{3}}(y)$.

Theorem 1. There are no finite metric bases for polyhedral gauges in $\mathbb{R}^{n}$.

Proof. Let us consider a polyhedral gauge $\gamma_{\mathcal{C}}$. Suppose that $B=\left\{b_{1}, \ldots, b_{k}\right\}$ is a metric basis for $\left(\mathbb{R}^{n}, d_{\mathcal{C}}\right)$. Considering a cone $c=(O, f)$ defined by a facet $f$ of $\mathcal{C}$ and the origin $O$, we denote $c_{1}, \ldots, c_{k}$ the cones defined by $c_{i}=c+\overrightarrow{O b_{i}}$. By lemma 5, we know that $\cap_{i=1}^{k} c_{i}$ is nonempty and unbounded. Therefore, there exist two points $x, y \in \cap_{i} c_{i}$ such that $g_{c_{1}}(x)=g_{c_{1}}(y)$. By lemma 6 , $x$ and $y$ have the same representation $r(x \mid B)=r(y \mid B)$, thus $B$ is not a resolving set for $\mathbb{R}^{n}$.

This theorem can be extended to any central-symmetric gauge partially polyhedral. Indeed, the existence of one hyperplanar facet is sufficient to define a cone in which no metric basis exists. 

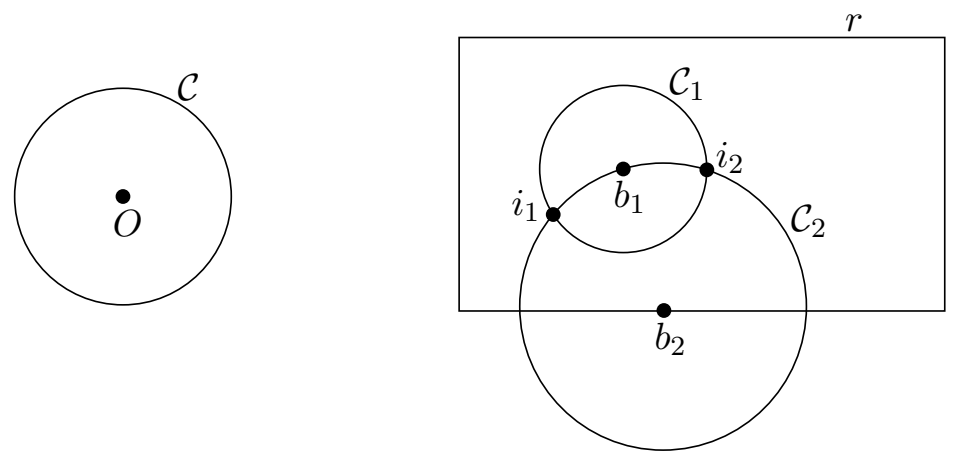

Fig. 3. Illustration for the proof of lemma 7 Left: the convex $\mathcal{C}$ defining the gauge $\gamma_{\mathcal{C}}$. Right: the balls $\mathcal{C}_{1}$ and $\mathcal{C}_{2}$ centered in $b_{1}$ and $b_{2}$ intersect one another in $i_{1}$ and $i_{2}$ inside the rectangle $r$.

Having at least two points which have the same distance from $O$ in a cone is a necessary condition for the theorem 1, This is not the case for all polyhedral gauges in $\mathbb{Z}^{n}$; indeed, if for any radius, each facet intersects a single point of $\mathbb{Z}^{n}$ then the metric dimension may be finite. This case may be detailed in an extended version of this paper. This condition is never met for chamfer norms; indeed, in each simplicial cone for a sufficiently large radius, the facet will intersect at least two points of $\mathbb{Z}^{n}$, because its normal has rational coordinates. Hence the theorem 1 remains valid for chamfer norms in $\mathbb{Z}^{n}$.

\section{Metric Bases for Gauges in Axes-Parallel Rectangles}

We consider here either polyhedral and non-polyhedral gauges. Let $\gamma_{\mathcal{C}}$ be a central-symmetric gauge and $r$ be an axes-parallel rectangle in $\mathbb{R}^{2}$.

Lemma 7. If $B=\left\{b_{1}, b_{2}\right\}$ is a metric basis for $r$, then $b_{1}$ and $b_{2}$ are points of the frontier of $r$.

Proof. Consider $B=\left\{b_{1}, b_{2}\right\}$ a metric basis for $r$, and suppose that $b_{1}$ is an interior point of $r$. Then we fix $\lambda_{1}$ such that $\mathcal{C}_{1}=\left(\lambda_{1} \mathcal{C}+\overrightarrow{O b_{1}}\right) \in r$ and $b_{2} \notin \mathcal{C}_{1}$. Let $\lambda_{2}=d_{\mathcal{C}}\left(b_{1}, b_{2}\right)$ and $\mathcal{C}_{2}=\left(\lambda_{2} \mathcal{C}+\overrightarrow{O b_{2}}\right)$. Hence the intersection $\partial\left(\mathcal{C}_{1}\right) \cap \partial\left(\mathcal{C}_{2}\right)$, where $\partial(A)$ is the border of $A$, results in two distinct points $i_{1}$ and $i_{2}$ in $r$. Finally $r\left(i_{1} \mid B\right)=r\left(i_{2} \mid B\right)$, so $B$ is not a resolving set for $\left(r, d_{\mathcal{C}}\right)$.

The lemma 7 is not valid in $\mathbb{Z}^{n}$. In fact, the intersection of discrete balls in $\mathbb{Z}^{n}$ might be slightly different from its continuous counterpart, resulting in less or more than two points. See section 5 for examples.

Lemma 8. Suppose that $\gamma_{\mathcal{C}}$ is a grid-symmetric gauge. If $\mathcal{C}$ does not contains any vertical nor horizontal facet, then the metric dimension of $\left(r, d_{\mathcal{C}}\right)$ is 2. 

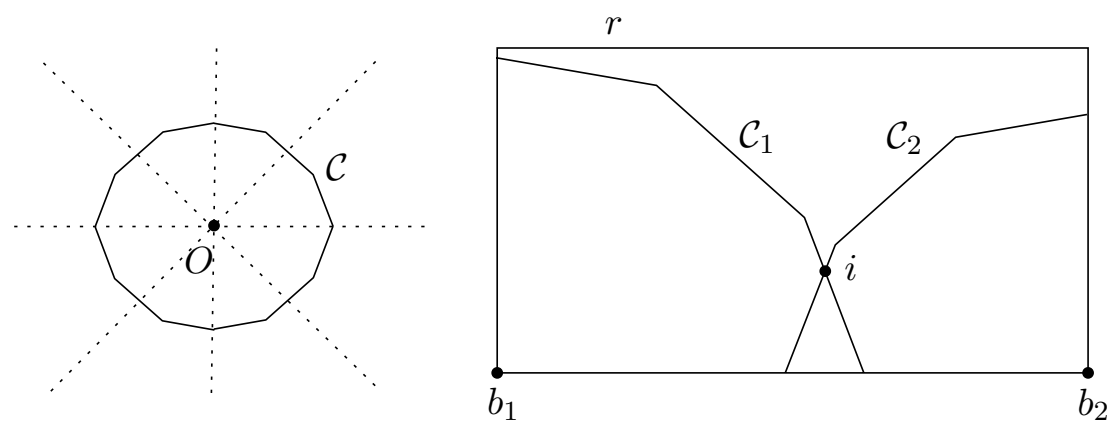

Fig. 4. Illustration of lemma 8 Left: a grid-symmetric gauge and its unit ball $\mathcal{C}$. Right: a single intersection $i$ between two balls $\mathcal{C}_{1}$ and $\mathcal{C}_{2}$ centered in $b_{1}$ and $b_{2}$.

Proof. The dimension is clearly $>1$. Fix $b_{1}$ and $b_{2}$ such that $b_{1}$ is the bottom-left corner of $r$ and $b_{2}$ is the bottom-right corner (See Fig. 4). For any $\lambda_{1}>0$, the intersection $\left(\lambda_{1} \mathcal{C}+\overrightarrow{O b_{1}}\right) \cap r$ is a monotonic decreasing curve, because the gauge is convex and grid-symmetric. For the same reasons, $\forall \lambda_{2}>0,\left(\lambda_{2} \mathcal{C}+\overrightarrow{\mathrm{Ob}_{2}}\right) \cap r$ is monotonic increasing. If we add the fact that $\mathcal{C}$ does not contain any vertical or horizontal facet, then we have both strictly monotonic curves. Intersection between strictly monotonic increasing and decreasing curves is at most a single point, thus $\left\{b_{1}, b_{2}\right\}$ is a resolving set for $\left(r, d_{\mathcal{C}}\right)$.

Corollary 1. The metric dimension for all Minkowski distances except $d_{\infty}$ is 2 in a rectangle.

Proof. $d_{\infty}$ is the only Minkowski distance whose balls contain vertical or horizontal facets.

Lemma 9. Let $\gamma_{\mathcal{C}}$ be a polyhedral gauge of metric dimension 2 in a rectangle $r$, $f i x\left\{b_{1}, b_{2}\right\}$ a metric basis of $\left(r, d_{\mathcal{C}}\right)$ such that $b_{1}$ is not a vertex of $r$, and denote $e_{1}$ the edge of $r$ containing $b_{1}$. Then $b_{2} \notin e_{1}$.

Proof. A small enough factor $\lambda_{1}>0$ exists, such that $\mathcal{C}_{1}$ intersects $e_{1}$ in two points $i_{1}$ and $i_{2}$, and such that $b_{2} \notin\left[i_{1}, i_{2}\right]$. Suppose that $i_{1} \in\left[b_{2}, i_{2}\right]$ and consider the cone $c_{1}$ defined by $b_{1}$ and a facet of $\mathcal{C}$ which contains $i_{2}$. Denote $c_{2}$ the cone centered in $b_{2}$ which contains the cone $c_{1}$ (see Fig. 51). So we have $\forall y, z \in c_{1}, g_{c_{1}}(y)=g_{c_{1}}(z) \Rightarrow g_{c_{2}}(y)=g_{c_{2}}(z)$. In consequence, $B$ cannot be a metric basis in the rectangle.

\section{Results and Discussion}

We have developed a program which gives by enumeration all metric bases for any given chamfer norm in an axes-parallel rectangle. The figure 6 shows for $d_{1}$ and the chamfer norms $\langle 3,4\rangle$ and $\langle 5,7,11\rangle$, the union of all metric bases vertices (in grey) in a $20 \times 12$ rectangle. As we can see, the vertices of a metric basis for 

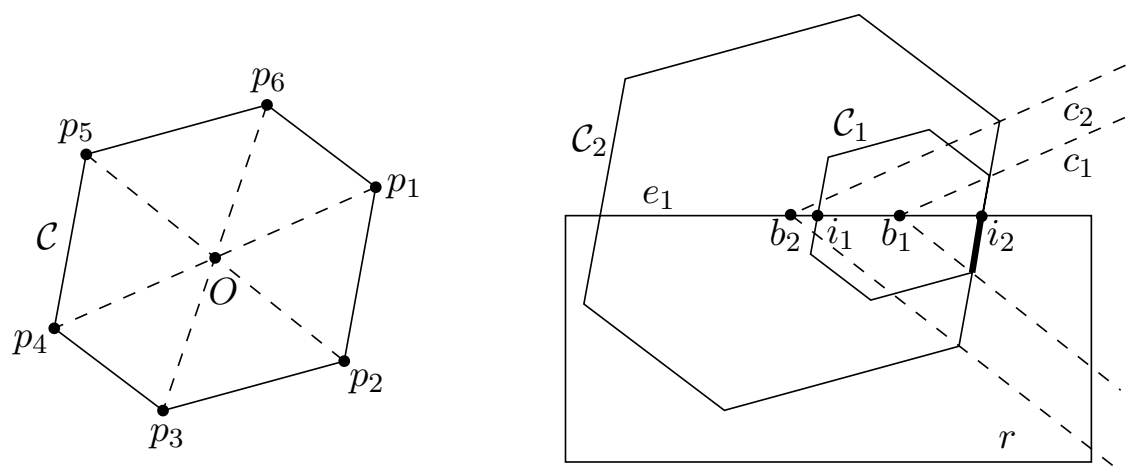

Fig. 5. Illustration of lemma 9 Left: A polyhedral gauge and its unit ball $\mathcal{C}$. Right: The bold line segment shows the intersection between the two balls $\mathcal{C}_{1}$ and $\mathcal{C}_{2}$ in $r$.

$d_{1}$ are necessarily located on the corners of the rectangle, while for $\langle 3,4\rangle$ they can be choosen in the first or second border inside the rectangle, and for the last case $\langle 5,7,11\rangle$, in the whole rectangle. This shows that lemma 7 does not always hold in $\mathbb{Z}^{n}$.

We also developed an interactive program which displays, for a set $S$ of vertices in a rectangular region $W$, the resolved and unresolved points (resp. in black, white and grey). Results are presented in Fig. [7] each column shows a configuration of two or three vertices; each row corresponds to a distance. The last distance is the chamfer norm $\langle 3,4,6\rangle$, obtained by replacing the corresponding weights in the mask $\langle 5,7,11\rangle$. The interest of this norm is that its balls are octogons with horizontal and vertical facets.

A point is said resolved in $W$ if its representation for $S$ is unique in $W$, while it is unresolved if there exists at least one other point in $W$ sharing the same representation. A set $S$ is then a resolving set for $W$ if all points of $W$ are resolved. In our example, only two of the 18 cases are resolving sets: rows (a) and (e) in the right column.

Now consider an axes-parallel subrectangle $R$ in $W$, such that the black vertices lie on the corners of $R$. If all the points in $R$ are resolved, then the set $S$ of vertices is a resolving set for $R$. This is the case for (a,b,d,e)-right.
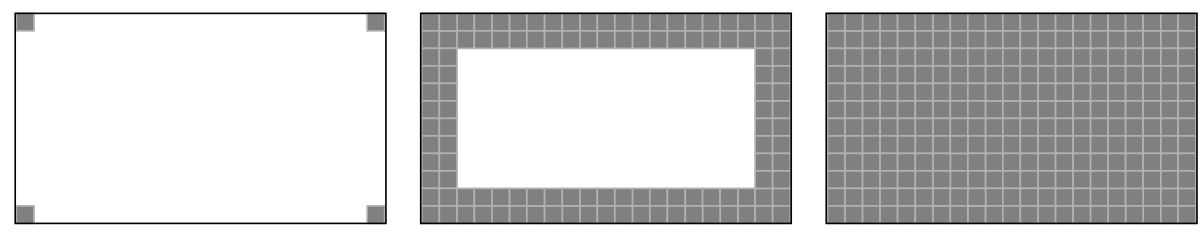

Fig. 6. Left to right: $d_{1},\langle 3,4\rangle,\langle 5,7,11\rangle$ 
(a)
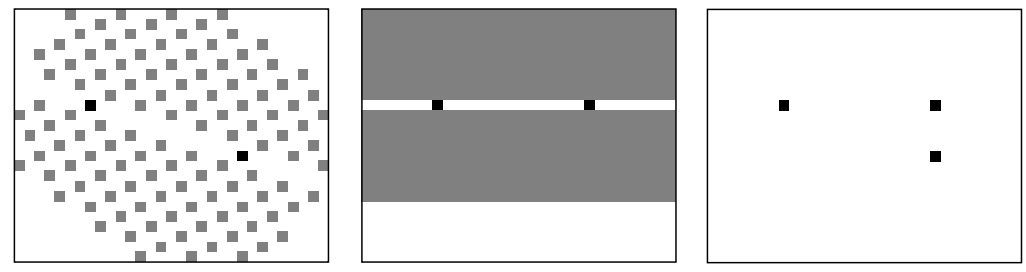

(b)
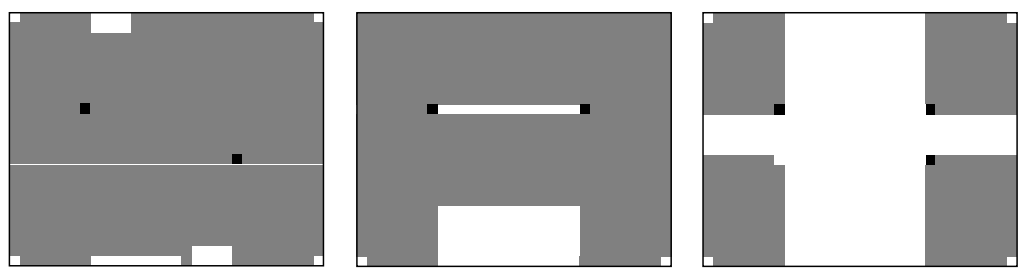

(c)
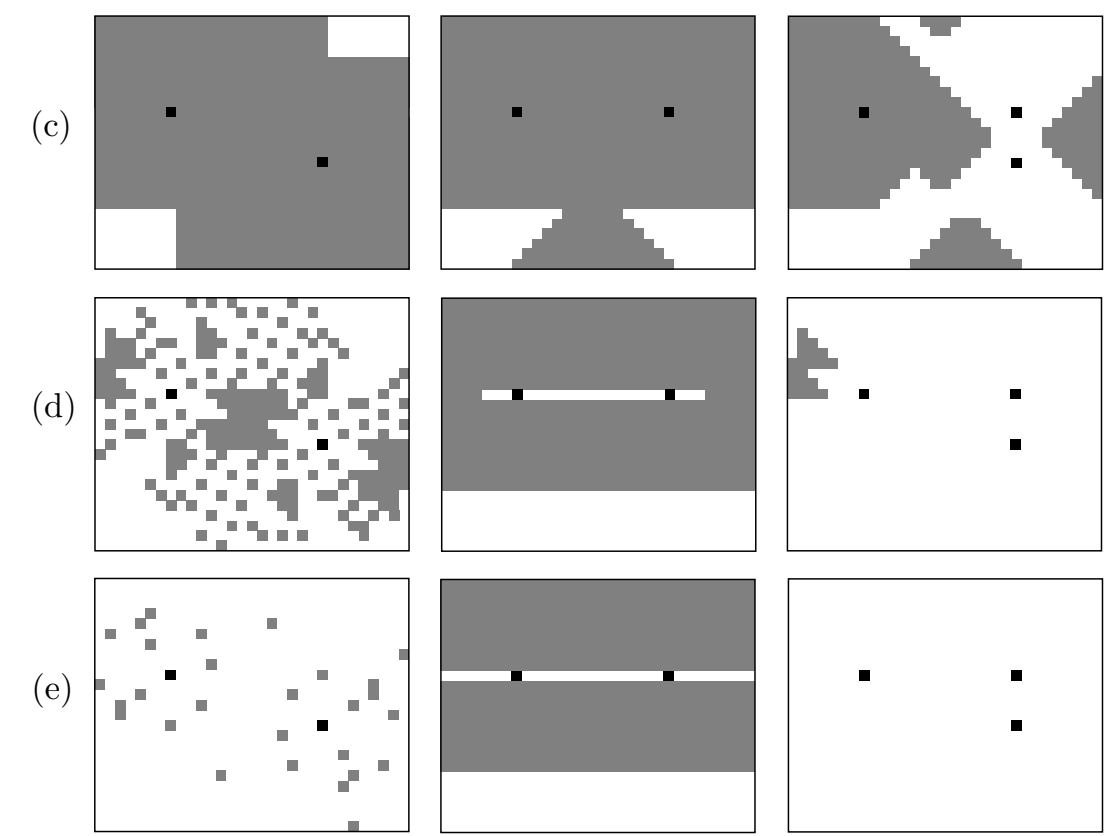

(f)
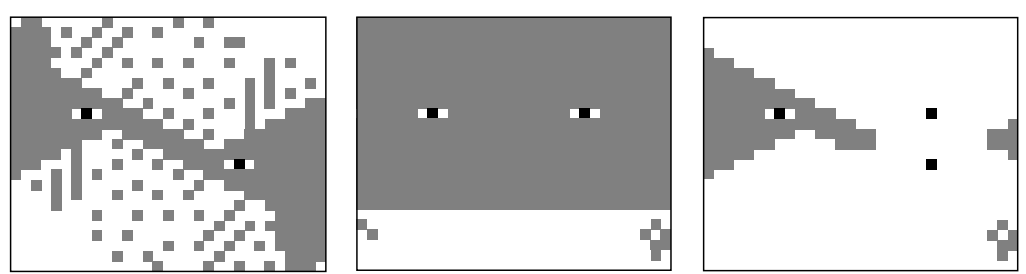

Fig. 7. Configurations for two or three vertices in a $31 \times 25$ rectangular region, using distances (a) $d_{2}$, (b) $d_{1}$, (c) $d_{\infty}$, (d) $\langle 3,4\rangle$, (e) $\langle 5,7,11\rangle$, (f) $\langle 3,4,6\rangle$. The vertices are shown in black, resolved points in white, unresolved points in grey. The coordinates of vertices from the origin $(0,0)$ at top left are $(7,9),(22,9)$ and $(22,14)$. 
In some other cases, $S$ is still a resolving set for $R$ since each unresolved point in $R$ has its equivalent points (points sharing the same representation, not represented here) lying outside $R$. We have this situation for (e)-left and (a,b,d,e)-middle, but not for (c,f)-middle. This can be explained by lemma 8 . since the balls of $d_{\infty}$ and $\langle 3,4,6\rangle$ have horizontal and vertical facets.

These different configurations are resulting from intersections of the cones in the distance balls. A thorough geometrical and arithmetical study might be a natural continuation of this paper for better understanding.

\section{Conclusion}

We have shown that the metric dimension of any polyhedral (or partially polyhedral) central symmetric gauge is infinite in $\mathbb{R}^{n}$, and for $\mathbb{Z}^{n}$ in the case of chamfer norms, whereas it is finite in axes-parallel rectangles. In the latter case, we have exhibited gauges having metric dimension 2 and we have completely characterized their metric bases. In the future, we aim at studying how to generalize our results for continuous and discrete gauges in rectangles, in convex or non-convex simple polyhedrons with direct or geodesic distances; showing the conditions where discrete polyhedral gauges may have finite basis in $\mathbb{Z}^{n}$, and finally, tackling the linked problems of forcing subsets and partitions dimension.

\section{References}

1. Berger, M.: Géométrie. Nathan (1990)

2. Blumenthal, L.M.: Theory and applications of distance geometry. Chelsea publishing company, New York (1970)

3. Buczkowski, P.S., Chartrand, G., Poisson, C., Zhang, P.: On $k$-dimensional graphs and their bases. Periodica Mathematica Hungarica 46, 9-15 (2003)

4. Cáceres, J., Hernando, C., Mora, M., Pelayo, I.M., Puertas, M.L.: On the metric dimension of infinite graphs. Electronic Notes in Discrete Math. 35, 15-20 (2009)

5. Chartrand, G., Eroh, L., Johnson, M.A., Oellermann, O.R.: Resolvability in graphs and the metric dimension of a graph. Discrete Applied Math. 105(1-3), 99-113 (2000)

6. Chartrand, G., Salehi, E., Zhang, P.: On the partition dimension of a graph. Congressus Numerantium 131, 55-66 (1998)

7. Chartrand, G., Salehi, E., Zhang, P.: The partition dimension of a graph. Aequationes Mathematicae 59, 45-54 (2000)

8. Chartrand, G., Zhang, P.: The forcing dimension of a graph. Mathematica Bohemica 126(4), 711-720 (2001)

9. Harary, F., Melter, R.A.: On the metric dimension of a graph. Ars Combinatoria 2, 191-195 (1976)

10. Hardy, G.H., Wright, E.M.: An introduction to the theory of numbers, 5th edn. Oxford Science Pub. (1979)

11. Hernando, C., Mora, M., Pelayo, I.M., Seara, C., Cáceres, J., Puertas, M.L.: On the metric dimension of some families of graphs. Electronic Notes in Discrete Mathematics 22, 129-133 (2005) 
12. Hulin, J.: Axe médian discret: propriétés arithmétiques et algorithmes. Thèse de Doctorat, Aix-Marseille Université (November 2009), http://pageperso.lif. univ-mrs.fr/ jerome.hulin/these-hulin.pdf

13. Khuller, S., Raghavachari, B., Rosenfeld, A.: Landmarks in graphs. Discrete Applied Mathematics 70(3), 217-229 (1996)

14. Melter, R.A., Tomescu, I.: Metric bases in digital geometry. Computer Vision, Graphics, and Image Processing 25(1), 113-121 (1984)

15. Normand, N., Evenou, P.: Medial axis lookup table and test neighborhood computation for 3D chamfer norms. Pattern Recognition 42(10), 2288-2296 (2009)

16. Slater, P.J.: Leaves of trees. Congressus Numerantium 14, 549-559 (1975)

17. Thiel, E.: Géométrie des distances de chanfrein. Habilitation à Diriger des Recherches, Université de la Méditerranée, Aix-Marseille 2 (December 2001), http://pageperso.lif.univ-mrs.fr/ edouard.thiel/hdr

18. Tomescu, I.: Discrepancies between metric dimension and partition dimension of a connected graph. Discrete Mathematics 308(22), 5026-5031 (2008)

19. Wikipedia: Gallery of named graphs, http://en.wikipedia.org/wiki/Gallery_ of_named_graphs 